\title{
Social Relations and PTSD Symptoms: A Prospective Study on Earthquake-Impacted Adolescents in Taiwan
}

\author{
Chih-Hsun Wu, Sue-Huei Chen, Li-Jen Weng, and Yin-Chang Wu \\ Department of Psychology, National Taiwan University, Taipei, Taiwan
}

\begin{abstract}
This prospective longitudinal study examined two competing models, a traditional social support model and a supportive and detrimental social relations model, to clarify the association of PTSD symptoms with supportive and detrimental social relations. Seven-hundred five adolescents living near the epicenter of the Taiwan Chi-Chi Earthquake participated in the study. The models were evaluated and cross-validated using structural equation modeling. The supportive and detrimental social relations model appeared to be a better fit. After further evaluation of three nested versions of the supportive and detrimental social relations model, detrimental social relations was found to partially mediate the relationship between PTSD symptoms 1 and 2 years following the earthquake. The findings suggest that helping adolescents deal with detrimental social relations can contribute to postdisaster adjustment.
\end{abstract}

Social support is one of the most important protective factors for coping with trauma. Two recent meta-analysis studies examining the risk/protective factors related to posttraumatic stress disorder (PTSD) revealed social support to be among the strongest predictive factors of PTSD (Brewin, Andrews, \& Valentine, 2000; Ozer, Best, Lipsey, \& Weiss, 2003). Trauma studies focusing on children and adolescents have also found that lack of social support constitutes a major risk factor for psychopathology (Pine \& Cohen, 2002). These results suggest that social support may serve as a buffer during and after trauma and that those with more social support will have less psychological distress afterwards.

As Kaniasty (2005) pointed out, one of the major problems is that some widely used social support models (e.g., buffering model, direct effect model) assume that social support is not directly influenced by stress; however, this does not appear to be the case in most trauma studies. Several studies have indicated that social support was likely to deteriorate after large-scale trauma (e.g., Hobfoll \& Stephens, 1990; Kaniasty, Norris, \& Murrell, 1990). To clarify this issue, Kaniasty and Norris (2008) conducted a 2year longitudinal study after the flood and mudslide in Mexico and examined the relation between perceived social support and PTSD symptoms. The study collected four waves of data at 6 (W1), 12 (W2), 18 (W3), and 24 (W4) months after the trauma. They found that at the earlier phase, W1 social support significantly predicted W2 PTSD, whereas W1 PTSD did not predict W2 social support. These results were consistent with the social causation theory (Dohrenwend, 2000) that claims the lack of social supports is an antecedent of psychological distress. At the middle phase, W2 social support and PTSD were found to significantly predict W3 PTSD and social support, respectively, revealing a reciprocal interaction. At the latter phase, W3 PTSD significantly predicted W4 social support, whereas W3 social support could not predict W4 PTSD. The findings at the latter phase were similar to the results found in a longitudinal study on veterans (King, Taft, King, Hammond, \& Stone, 2006). Their results also showed that initial PTSD symptoms had a significant negative relation with perceived social support 5 years later. However, the initial perceived social support did not reliably predict later PTSD symptoms. These results supported the notion of social selection theory (Dohrenwend, 2000), which hypothesizes that psychological reactions (for example, anger outbursts or depression) may result in diminished perceived support from others or alternatively, actually jeopardize relations with others. Taken together, these results support a transactional relationship between social support and PTSD symptoms.

Following the study noted above, the current study examined another important issue in regard to social support in trauma research, i.e., the relationship between negative social interactions and PTSD symptoms (Guay, Billette, \& Marchand, 2006). As stated earlier, lack of social support is harmful in regard to coping with trauma. However, we hypothesized that there may be detrimental effects associated with experiencing negative social interactions while expecting to benefit from social support. As well as being supportive, interpersonal relationships may also function

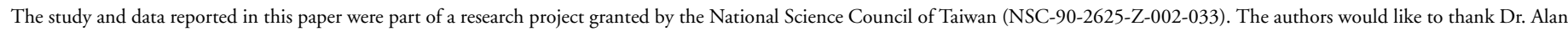

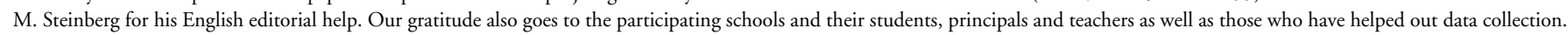

Correspondence concerning this article should be addressed to: Sue-Huei Chen, Department of Psychology, National Taiwan University, Taipei, 106, Taiwan. E-mail: shchen@ntu.edu.tw.

(C) 2009 International Society for Traumatic Stress Studies. Published online in Wiley InterScience (www.interscience.wiley.com) DOI: 10.1002/jts.20447 
as a source of conflict, strain, and disappointment (Rook, 1998). Evidence suggests that negative social interactions may have a stronger association with well-being than positive social interactions (e.g. Schuster, Kessler, \& Aseltine, 1990). Similar results have been found in trauma studies, in that positive social support had a lesser impact on PTSD than negative social interactions (Andrews, Brewin, \& Rose, 2003; Zoellner, Foa, \& Bartholomew, 1999). The issue of posttrauma negative social relations may be even more salient among adolescents. For example, Pynoos, Steinberg, and Wraith (1995) proposed a developmental model of childhood traumatic stress in which subsequent trauma reminders and secondary stresses and adversities play an important role in relation to posttrauma distress and developmental psychopathology. In this model, negative social relations, especially among peers, serves as a major source of secondary stress for adolescents.

The current study examined this issue by using structural equation modeling (SEM) to compare the two competitive models in a longitudinal study. First, a traditional social support model, following a unidimensional conception of social support, proposes that positive and negative social relations represent the two ends of the same latent social support construct. Based on the traditional social support model (as shown in Figure 1a), PTSD symptoms during the first year after the trauma (PTSD1) affect social support and PTSD symptoms of the second year (PTSD2), and PTSD2 is also affected by social support. Second, the supportive and detrimental social relations model proposes that detrimental (negative) social relations and supportive (positive) social relations are affected by PTSD1 and then affect PTSD2 in their own specific ways (as shown in Figure 1b).
Moreover, to evaluate possible associations, further clarification of the roles of social relations was attempted by using three nested models of the supportive and detrimental social relations model. The first model (Figure 2a) hypothesizes that there is no association (total mediation) between PTSD1 to PTSD2 once the effect of social relations has been taken into account. The second model (Figure $2 \mathrm{~b}$ ) tests the notion of social causation theory, and hypothesizes that PTSD will not influence supportive social relations and detrimental social relations. The third model (Figure2c) tests the notion of social selection theory, and hypothesizes that supportive social relations and detrimental social relations will not affect PTSD. We developed these models to help us verify if detrimental social relations play a specific role in the course of PTSD symptoms after trauma.

For most people, PTSD symptoms usually remit within a year after trauma (Norris et al., 2002). Thus, after clarifying the role of detrimental social relations, we examined whether the aboveverified paths between PTSD symptoms and social relations would manifest different patterns between those who could be diagnosed with and without PTSD over an amount of time (one year in the current study) after trauma.

In sum, under the hypothesis that detrimental social relationships might play a significant role in maintaining PTSD symptoms, this study had three specific aims. The first was to test the hypothesis that detrimental social relations have specific pathways of influence on PTSD by comparing two models, the traditional social support model and the supportive and detrimental social relations model. Second, if the supportive and detrimental social relations model proved acceptable, three nested models would be

a. The TSS model

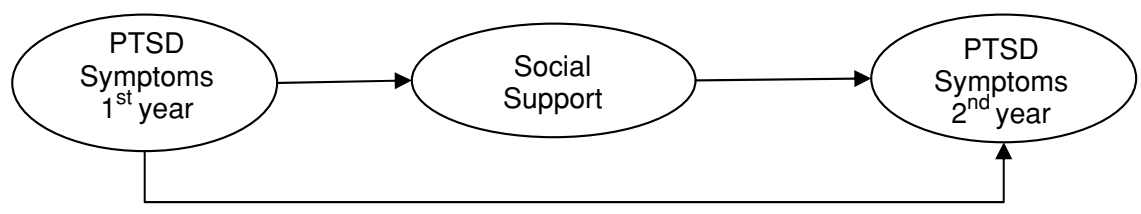

b. The SDSR model

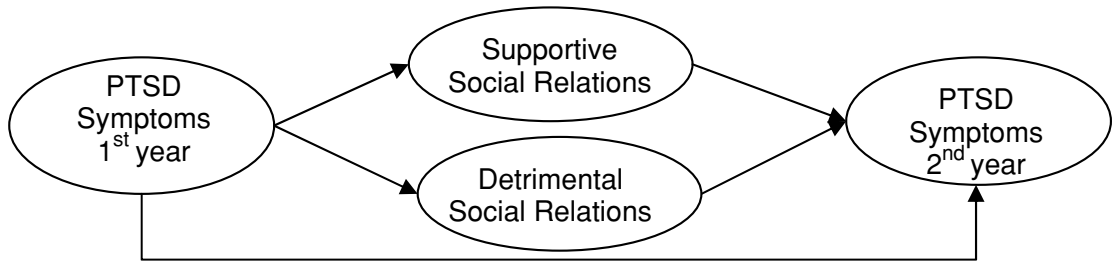

Figure 1. The conceptual framework of the two competitive models: (a) the traditional social support (TSS) model; (b) the supportive and detrimental social relations (SDSR) model. 
a. Examining Total Mediation

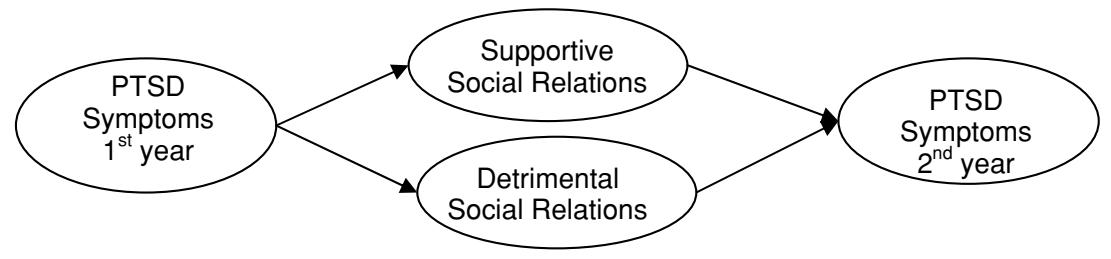

b. Examining Social Causation

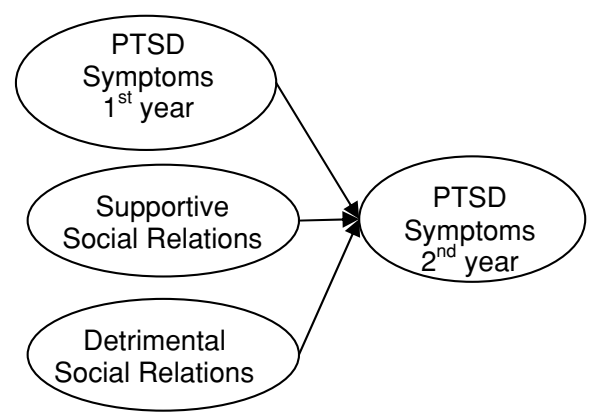

c. Examining Social Selection

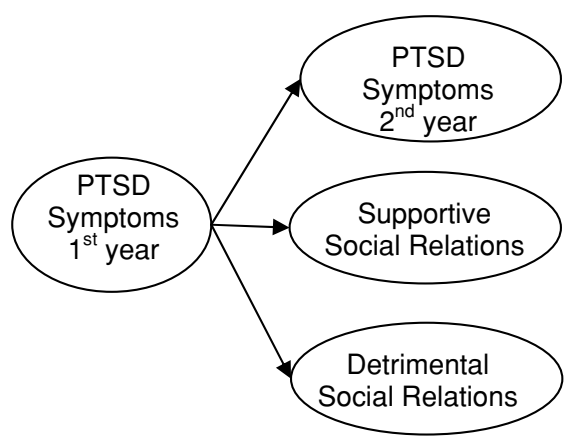

Figure 2. The conceptual framework of the three nested models of the supportive and detrimental social relations model: (a) the total mediation model; (b) the social causation model; (c) the social selection model.

evaluated to further clarify the role of detrimental social relations. Third, it is hypothesized that the paths linking PTSD symptoms with detrimental and supportive social relations will be different between subclinical and nonclinical adolescents. We used the multigroup method of SEM to examine it.

\section{METHOD}

\section{Participants}

The Taiwan 921 Chi-Chi Earthquake (measured 7.3 on the Richter scale) occurred on September 21, 1999. Approximately 2,500 people died and more than 11,000 were injured. Thousands of houses and school buildings collapsed or suffered significant damage (National Alliance for Post-Earthquake Reconstruction, 2000). A large number of children and adolescents suffered from posttraumatic stress symptoms 1-2 years after the event (Chen, Lin, Tseng, \& Wu, 2002). This study uses data from a largescale postearthquake prospective study, which was approved by the National Science Council of Taiwan.

The study took place in two towns located within the perimeter of the earthquake epicenter, which had the highest rates of earthquake-related mortality, morbidity, and damage in Taiwan. Each of the two towns has three junior high schools, and the participants of this study came from two junior high schools in each town. Participants were selected in 2000 as follows: (a) In the two smaller schools (which have less than five classes in each grade), all students in the seventh and eighth grade were selected; (b) In the two larger schools, three to four classes of students were randomly selected from the seventh and eighth grades. All were followed up in 2001 when they became eighth and ninth graders. In Taiwan, the same-age students were randomly assigned to each class of their district school. Thus, the distribution and earthquake exposure of the participating students of these two particular towns were supposed to be similar. Prior to starting the study, the research sponsor, the National Science Council of Taiwan, as well as all four school boards (including principal, teachers, and parent representatives) approved the study plan. A passive consent form was given to students and their parents. With the support of the classroom teachers, all measures were given to the students in their usual classrooms.

The data for the first year, including demographic data and PTSD scores, were collected during the first 2 weeks of September of 2000. The second-year data, including PTSD scores and scores from the Taiwan Relationship Inventory for Children and Adolescents-short version, were collected from September through October of 2001. After eliminating those who had provided unusable data, 790 adolescents completed the first-year data collection, consisting of $46.2 \%$ girls $(n=365)$ and $53.8 \%$ boys $(n=425)$. For the second-year data collection, 38 female and 47 male adolescents dropped out, leaving a follow-up rate of $89.2 \%$. Seven-hundred five adolescents completed both waves of data collection and made 
up the final sample, consisting of $46.4 \%$ girls $(n=327)$ and $53.4 \%$ boys $(n=378)$.

\section{Measures}

The UCLA PTSD Reaction Index for DSM-IV, Revision 1, Adolescent Version (Steinberg, Brymer, Decker, \& Pynoos, 2004) in traditional Chinese (Chen et al., 2002) is a self-report questionnaire. Following the diagnostic classification system for PTSD in the Diagnostic and Statistical Manual of Mental Disorders, Fourth Edition (DSM-IV; American Psychiatric Association, 1994), traumatic experiences and their objective features (Criterion A1) and subjective responses during or right after the trauma (Criterion A2) were first evaluated. Then, posttraumatic stress reactions were evaluated with a 22 -item symptom checklist. The checklist is composed of three major parts: reexperiencing (Criterion B; five items), avoidance/numbing (Criterion $\mathrm{C}$; nine items), and increased arousal (Criterion D; six items). Participants were asked to rate the frequency of the PTSD symptoms experienced during the past month on a 5-point Likert scale ranging from 0 (not at all) to 4 (most of the time). Scoring algorithms and diagnostic screening procedures for the Reaction Index have been previously described (Steinberg et al., 2004). Those who met all the criteria (A1, A2, $\mathrm{B}, \mathrm{C}$, and $\mathrm{D})$ were classified as "DSM-IV full PTSD diagnosis likely." Those who met Criterion $\mathrm{A}$ as well as meeting criteria in two of the three B, C, and D categories were "partial PTSD diagnosis likely." This procedure was employed by Pfefferbaum and colleagues (Pfefferbaum, Stuber, Galea \& Fairbrother, 2006) in their study of PTSD after the terrorist attacks on the World Trade Center. The PTSD Reaction Index has good psychometric properties (Steinberg et al., 2004) and has been used in Taiwan in several studies (Chen et al., 2002; Chen \& Wu, 2006).

The Taiwan Relationship Inventory for Children and Adolescents, Short Version (TRICA-S) was modified from the original Taiwan Relationship Inventory for Children and Adolescents (Wu, Wu, Hsu, \& Shiau, 2008). The 36-item TRICA-S has three subscales: parents, teachers, and peers. The parents subscale of the TRICA-S is composed of two factors: supportive relationships with parents (eight items) and detrimental relationships with parents (eight items). Examples of the items include "When you feel sad or scared, your mother will figure out a way to help you" and "Your father is very picky about anything you do" for supportive and detrimental relationships with parents, respectively. Similar 2-factor structures can be seen in both the teachers and peers subscales, i.e., supportive and detrimental relationships with teachers (five items each) as well as supportive and detrimental relationships with peers (five items each), respectively. The participants were asked to rate the frequency of each statement on a 4-point Likert scale ranging from 1 (never) to 4 (most of the time). All six factors have satisfactory reliabilities, with internal consistency Cronbach's alpha ranging from .73 to .85 .

\section{Model Specification}

The traditional social support model was composed of three latent variables: PTSD1, PTSD2, and social support. The latent constructs of PTSD1 had three indicators: reexperiencing first year, avoidance first year, and increased arousal first year, using the scores of the Criterion B, C, D scores of the PTSD Criterion A-C, respectively. Similarly, PTSD2 had three indicators: reexperiencing second year, avoidance second year, and increased arousal second year. The latent construct of social support was composed of six indicators: supportive and detrimental relationships with parents, with teachers, and with peers, using the corresponding factor scores from Taiwan Relationship Inventory for Children and Adolescents-Short Version. The structural model of the traditional social support model included three paths: from PTSD1 to social support and to PTSD2, and from social support to PTSD2. The error covariances between Re-experiencing first and second year, Avoidance first and second year, and Increased Arousal first and second year were evaluated due to using the same measurement items. The error covariances between supportive and detrimental relationships with parents, supportive and detrimental relationships with teachers, and supportive and detrimental relationships with peers were evaluated due to rating the same relational source.

The supportive and detrimental social relations model was composed of four latent variables: PTSD1, PTSD2, supportive social relations, and detrimental social relations. The measurement model of PTSD1 and PTSD2 was the same as in the traditional social support model. The latent construct of supportive social relations as well as detrimental social relations were both composed of three indicators: supportive relationships with parents, with teachers, and with peers; as well as detrimental relationships with parents, with teachers, and with peers, respectively. The structural model of the supportive and detrimental social relations model has included five paths: from PTSD1 to supportive social relations, to detrimental social relations, and to PTSD2, and from supportive social relations and detrimental social relations to PTSD2. The six error covariances were the same as the traditional social support model.

\section{Data Analusis}

All 705 participants reported that the earthquake was their most traumatic lifetime experience. Based on the participants' first year results on the diagnostic evaluation of PTSD, 55 adolescents met the criterion for DSM-IV full PTSD diagnosis likely, and 111 adolescents met the criterion for partial PTSD diagnosis likely (10 met criteria $\mathrm{B}+\mathrm{C}$; 92 met criteria $\mathrm{B}+\mathrm{D} ; 9$ met criteria $\mathrm{C}+\mathrm{D})$. These students were then selected to form the subclinical group $(n=$ $166,51.2 \%$ were female). Five hundred thirty-nine adolescents, who did not meet diagnostic criteria, were randomly assigned into two groups, the Nonclinical Group $1(n=272,44.5 \%$ were female) and Nonclinical Group $2(n=267,45.3 \%$ were female $)$, 
Table 1. Demographic Variables, Posttraumatic Stress Disorder (PTSD) Symptoms and Social Relations Scores in Three Groups

\begin{tabular}{|c|c|c|c|c|c|c|}
\hline & \multicolumn{6}{|c|}{ Group } \\
\hline & \multicolumn{2}{|c|}{$\begin{array}{l}\text { Nonclinical } 1 \\
(n=272)\end{array}$} & \multicolumn{2}{|c|}{$\begin{array}{c}\text { Nonclinical } 2 \\
(n=267)\end{array}$} & \multicolumn{2}{|c|}{$\begin{array}{l}\text { Subclinical } \\
(n=166)\end{array}$} \\
\hline & $M$ & $S D$ & $M$ & $S D$ & $M$ & $S D$ \\
\hline Age $(M, S D)$ & 13.32 & 0.83 & 13.36 & 0.82 & 13.27 & 0.82 \\
\hline \multicolumn{7}{|l|}{ PTSD Symptoms } \\
\hline Reexperience 1st year & 4.76 & 3.37 & 4.74 & 3.17 & 11.46 & 4.17 \\
\hline Avoidance 1st year & 5.97 & 3.93 & 5.71 & 4.26 & 12.89 & 5.31 \\
\hline Increased arousal 1st year & 6.38 & 3.01 & 6.22 & 3.13 & 12.33 & 3.07 \\
\hline Reexperience 2nd year & 3.69 & 3.56 & 3.43 & 3.17 & 5.89 & 4.69 \\
\hline Avoidance 2nd year & 4.87 & 4.80 & 4.62 & 4.22 & 7.75 & 5.78 \\
\hline Increased arousal 2nd year & 5.69 & 3.65 & 5.59 & 3.68 & 8.15 & 4.33 \\
\hline \multicolumn{7}{|l|}{ Social relations } \\
\hline Parents: Supportive & 19.50 & 5.09 & 19.67 & 5.19 & 19.38 & 5.30 \\
\hline Parents: Detrimental & 15.59 & 4.57 & 15.17 & 4.55 & 16.38 & 5.15 \\
\hline Teachers: Supportive & 13.23 & 3.23 & 13.24 & 3.38 & 13.46 & 3.48 \\
\hline Teachers: Detrimental & 9.45 & 3.41 & 9.29 & 3.28 & 10.14 & 3.60 \\
\hline Peers: Supportive & 14.55 & 3.09 & 14.78 & 3.13 & 15.10 & 3.13 \\
\hline Peers: Detrimental & 8.61 & 2.47 & 8.43 & 2.51 & 8.68 & 2.68 \\
\hline
\end{tabular}

for model evaluation and cross-validation, respectively. There were no significant differences on demographic characteristics among the three groups (Table 1).

The scores of all measured variables of the three groups are listed in Table 1. Prior to evaluating the models, examination of all measured variables showed no significant differences between the two nonclinical groups, indicating that these two samples could be used as equivalent samples. The skewness and kurtosis of all measured variables of the three groups were checked, indicating that the normal distribution hypothesis for SEM was not violated.

The SEM method was used to evaluate and compare the traditional social support model and supportive and detrimental social relations model. The LISREL (8.7; Joreskog \& Sorbom, 2004) was used to perform SEM. A covariance matrix was used as the data set, and the maximum likelihood method was used for model estimation. With regard to model evaluation, as suggested by Kline (2005), the following indices were used in the study: the model chi-square, root mean square error of approximation (RMSEA), Bentler comparative fit index (CFI), standardized root mean square residual (SRMR), goodness-of-fit index (GFI), and Akaike information criterion (AIC). It is suggested that SRMR $<.10$ and GFI $\geq .90$ (Kline, 2005), as well as RMSEA $\leq .06$ and CFI $\geq .95(\mathrm{Hu}$ $\&$ Bentler, 1999) indicate good overall fit of the model. The AIC index was used in nonnested model comparisons. A smaller AIC index indicates a better model (Kline, 2005). Cross-validation, using a parallel sample, was performed to validate the results. The z' statistics (MacKinnon, Lockwood, Hoffman, West, \& Sheets, 2002) were used to test the mediation effect. Changes of the chisquare value $\left(\Delta \chi^{2}\right)$ were used in further examination of the nested models. The traditional social support model and the supportive and detrimental social relations model were then evaluated for the subclinical sample to investigate acceptability of the two proposed models. Moreover, a multigroup method of SEM was used to evaluate the subclinical sample and nonclinical sample simultaneously to clarify if there were any differences on structural paths between the two samples.

\section{RESULTS}

\section{Evaluation and Comparison of the Traditional Social Support and Supportive and Detrimental Social Relations Models}

The data for Nonclinical Group 1 were used in model evaluation. As shown in Table 2, the traditional social support model did not fit the data. The GFI and CFI indices were too small; the RMSEA and SRMR indices were too large. The supportive and detrimental social relations model was acceptable, with all model fit indices indicating good overall fit. Additionally, the AIC 
Table 2. Model Fit Indices of TSS and SDSR Models in Three Groups

\begin{tabular}{lcccccccc}
\hline Sample & Model & $\chi^{2}$ & $d f$ & GFI & CFI & RMSEA & SRMR & AIC \\
\hline Nonclinical Group 1 & TSS & $186.24^{* *}$ & 45 & .89 & .89 & .11 & .13 & 263.73 \\
& SDSR & $78.01^{* *}$ & 43 & .96 & .97 & .05 & .06 & 142.04 \\
Nonclinical Group 2 & TSS & $170.43^{* *}$ & 45 & .89 & .88 & .11 & .12 & 254.37 \\
& SDSR & $67.73^{*}$ & 43 & .96 & .98 & .05 & .05 & 137.17 \\
Subclinical Group & TSS & $100.13^{* *}$ & 45 & .90 & .91 & .10 & .11 & 178.23 \\
& SDSR & 47.83 & 43 & .95 & .99 & .03 & .06 & 120.18 \\
\hline
\end{tabular}

Note. TSS $=$ Traditional social support model; SDSR $=$ supportive and detrimental social relations model; GFI $=$ goodness-of-fit index; CFI $=$ Bentler comparative fit index; RMSEA = root mean square error of approximation; SRMR = standardized root mean square residual; AIC = Akaike information criterion.

${ }^{*} p<.01 .{ }^{* *} p<.001$.

index of the supportive and detrimental social relations model was smaller than that of the traditional social support model. All results suggest acceptance of the supportive and detrimental social relations model.
The results of parameter estimations and standardized parameter solutions for the supportive and detrimental social relations model are presented in Figure 3. PTSD1 has positive effects on detrimental social relations and PTSD2. Detrimental social

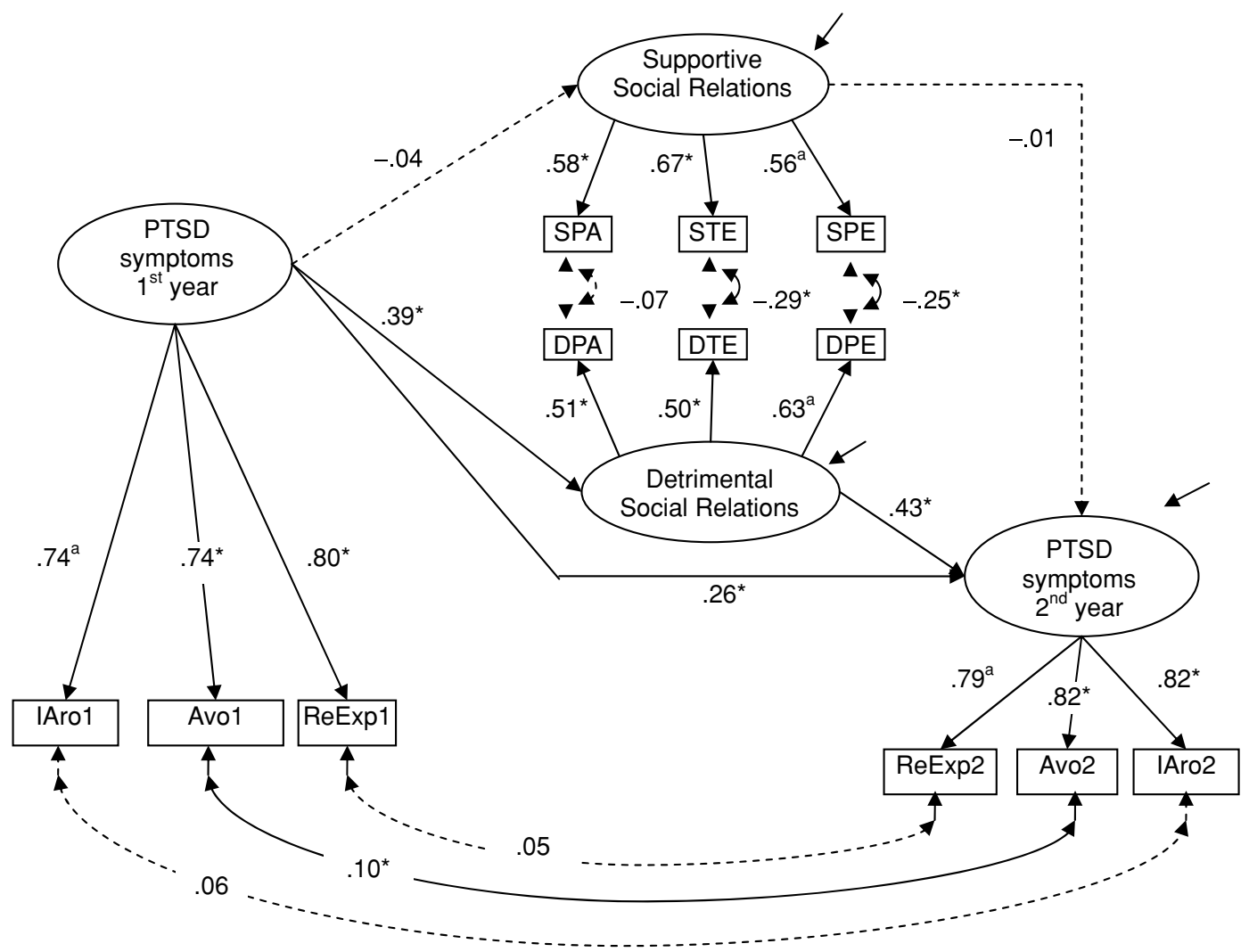

Figure 3. The supportive and detrimental social relations model with standardized parameter estimations for the Nonclinical Group 1. Note: *t $>1.96$; ${ }^{\text {a }}$ nonstandardized parameter fixed as 1 to fix the scale. ReExp $=$ Reexperience; Avo $=$ avoidance; IAro $=$ increased arousal; $\mathrm{SPA}=$ parents: supportive; DPA $=$ parents: detrimental; $\mathrm{STE}=$ teachers: supportive; $\mathrm{DTE}=$ teachers: detrimental; $\mathrm{SPE}=$ peers: supportive; DPE $=$ peers: detrimental. 
Table 3. Results of Examinations on the Three Nested Models of the SDSR Model

\begin{tabular}{llccc}
\hline Model examined & \multicolumn{1}{c}{ Constraint } & $\chi^{2}$ & $d f$ & $\Delta \chi^{2}$ \\
\hline Original SDSR model & No constraints & 78.01 & 43 & \\
Total mediation & PTSD $1 \rightarrow$ PTSD2 $=0$ & 86.46 & 44 & $8.45^{*}$ \\
Social causation & PTSD $\rightarrow$ SSR and DSR $=0$ & 96.85 & 45 & $18.84^{* *}$ \\
& PTSD $\rightarrow$ SSR $=0$ & 78.21 & 44 & $<1$ \\
Social selection & PTSD $\rightarrow$ DSR $=0$ & 96.80 & 44 & $18.79^{* *}$ \\
& SSR and DSR $\rightarrow$ PTSD2 $=0$ & 101.61 & 45 & $23.60^{* *}$ \\
& SSR $\rightarrow$ PTSD2 $=0$ & 78.03 & 44 & $<1$ \\
& DSR $\rightarrow$ PTSD2 $=0$ & 101.60 & 44 & $23.59^{* *}$ \\
\hline
\end{tabular}

Note. $\Delta \chi^{2}=\chi^{2}$ (each constrained condition) $-\chi^{2}$ (original SDSR model). SDSR $=$ Supportive and detrimental social relations model; PTSD1 $=$ posttraumatic stress disorder symptoms 1st year, PTSD2 $=$ PTSD symptoms 2 nd year; SSR $=$ supportive social relations; DSR = detrimental social relations.

${ }^{*} p<.01 .{ }^{* *} p<.001$.

relations also has positive effects on PTSD2. The paths from PTSD1 to supportive social relations and from supportive social relations to PTSD2 are not significant. The percentage of variance explained $\left(R^{2}\right)$ for PTSD2, supportive social relations, and detrimental social relations are $.34, .00$, and .15 , respectively. Taken together, the supportive and detrimental social relations model is accepted, suggesting that detrimental and supportive social relations have their own specific roles. Specifically, detrimental social relations serves as a partial mediator of PTSD symptoms $\left(z^{\prime}=\right.$ $2.91, p<.001)$, whereas supportive social relations does not have any significant influence in the model $\left(z^{\prime}=0.12, n s\right)$.

The data for the Nonclinical Group 2 were used for crossvalidation. The results consistently showed that the traditional social support model was rejected and that the supportive and detrimental social relations model was acceptable (see Table 2), again confirming acceptance of the supportive and detrimental social relations model.

\section{Examination of the Nested Models of the Supportive and Detrimental Social Relations Model}

Because the supportive and detrimental social relations model was accepted through model evaluation and cross-validation, further examination of the nested model was conducted. As shown in Table 3, the result of testing the total mediation model (Figure $2 \mathrm{a}$ ) yielded a significant increase on chi-square value, indicating that the link from PTSD1 to PTSD2 was significantly greater than zero. Similarly, the results of testing the social causation mechanism (Figure $2 \mathrm{~b}$ ) and social selection mechanism (Figure 2c) both yielded a significant increase of chi-square value. Further examination indicated that the link from PTSD1 to detrimental social relations as well as the link from detrimental social relations to PTSD2, respectively, was significantly greater than zero.

\section{Examination of the Subclinical Group}

Similar to the findings for the nonclinical group, the analyses using the subclinical group indicated that the supportive and detrimental social relations model was an acceptable model to interpret the subclinical phenomena, and the traditional social support model was still rejected (see Table 2).

By using the multigroup method of SEM to simultaneously evaluate two groups, the equality of the structural paths between Nonclinical Group 1 and the subclinical group were examined (see Table 4). While constraining all five structural paths to be equal between the two groups, the results yielded a significant increase of chi-square value as compared with the condition of no constraints, indicating that at least one of the five paths was not equal. Further examination by testing one path at a time showed that it was the link from detrimental social relations to PTSD2 that differed

Table 4. Examinations of Paths' Equality in the SDSR Model Between Subclinical Group and Nonclinical Group 1

\begin{tabular}{lccc}
\hline Equality constraints & $\chi^{2}$ & $d f$ & $\Delta \chi^{2}$ \\
\hline No constraints & 125.84 & 86 & \\
All five paths equal & 138.85 & 91 & $13.01^{* a}$ \\
Set PTSD1 $\rightarrow$ PTSD2 Free & 137.56 & 90 & $1.29^{\mathrm{b}}$ \\
Set PTSD1 $\rightarrow$ DSR Free & 136.55 & 90 & $2.30^{\mathrm{b}}$ \\
Set PTSD1 $\rightarrow$ SSR Free & 138.03 & 90 & $<1^{\mathrm{b}}$ \\
Set DSR $\rightarrow$ PTSD2 Free & 133.08 & 90 & $5.77^{* \mathrm{~b}}$ \\
Set SSR $\rightarrow$ PTSD2 Free & 137.92 & 90 & $<1^{\mathrm{b}}$ \\
\hline
\end{tabular}

Note. SDSR $=$ Supportive and detrimental social relations model; PTSD1 $=$ posttraumatic stress disorder symptoms 1st year; PTSD2 $=$ PTSD symptoms 2nd year; SSR = supportive social relations; DSR $=$ detrimental social relations.

${ }^{\mathrm{a}} \Delta \chi^{2}=\chi^{2}$ (all 5 paths equal) $-\chi^{2}$ (no constraints). ${ }^{\mathrm{b}} \Delta \chi^{2}=\chi^{2}$ (all 5 paths equal) $-\chi^{2}$ (each constrained condition).

$* p<.05$. 
significantly between the two groups. More specifically, the path value of detrimental social relations to PTSD2 was significantly greater for the subclinical group (1.85) as compared with the Nonclinical Group 1 (0.79).

\section{DISCUSSION}

The results of the current study support rejection of the traditional social support model and acceptance of the supportive and detrimental social relations model. Specifically, detrimental social relations served as a partial mediator of the changes in PTSD symptoms over time. Supportive social relations did not play a significant role.

Regarding the influence of PTSD symptoms on social support, Kaniasty and Norris (2008) found that the severity and persistence of PTSD might be related to the deterioration of social support. However, the current study found that PTSD symptoms contributed even more to adolescents' negative relations with others, rather than just lowering one's perceived social support. More specifically, it may be that some symptoms of PTSD, such as irritability and outbursts of anger, markedly diminished interest or participation in significant activities, feelings of detachment or estrangement from others, and restricted range of affect, are causally related to social rejection and social neglect among children and adolescents. Several studies have found that adolescents who showed more aggressive behaviors had a higher probability of rejection by their peers (e.g., Kupersmidt \& Coie, 1990). Other studies have also found that distress and lack of interactions with others may cause social rejection and/or social neglect (see Schneider, 2000, for a review). Thus, it is likely that PTSD symptoms increase the chance of having negative social interactions with others.

The results showed that negative social relations have a significant adverse impact on PTSD symptoms, suggesting that negative social relations may be construed as a type of secondary stress or adversity (Pynoos, et al., 1995). If we consider negative social relations as a secondary stress, the results of the current study can be conceptualized in terms of PTSD symptoms being exacerbated by an important source of secondary stress. Moreover, negative social relations may make it more difficult for adolescents to benefit from available social resources. Thus, a possible dual role of negative social relations might help to explain why it has a stronger impact on PTSD symptoms than positive social relations.

The results of the comparisons of the nonclinical and subclinical groups may be seen to further the consideration of negative social relation as a type of secondary stress. Quite interestingly, the only significant difference between the two groups was found on the path from detrimental social relations to PTSD2, which was greater in the subclinical group than in the nonclinical group. The paths directly from PTSD1 to PTSD2 and to detrimental social relations were not significantly different between the two groups. This means that detrimental social relations is more likely to predict the PTSD symptoms in the following year for those adolescents with likely or partially diagnosed PTSD in the first year, as compared with nonclinical participants. It may further suggest that the subclinical participants cope poorly with negative social relations. As Rook (2003) proposed, several factors such as lower self-esteem and less satisfying friendships and family relationships may influence reactivity to negative social exchange. Thus, a question may arise as to whether having subclinical PTSD may induce poorer reactions to detrimental social relations and thus extend PTSD symptoms afterwards. Further studies are needed to clarify this issue.

Moreover, there might be cultural reasons supporting the significance of negative social relations. In Yang's (1981) review, the author notes that Taiwanese culture is highly socially oriented. Attempting to achieve harmony and social acceptance, and avoiding social conflict, rejection, and embarrassment are extremely important. Thus, we may speculate that Taiwanese adolescents appraise negative social relations as a serious stress, especially when these negative relations are with someone they care about. This idea could be supported by a recent Taiwanese study (Yeh \& Lay, 2008), in that Taiwanese fourth and sixth graders showed more vulnerability in response to negative verbal provocations delivered by liked as opposed to disliked peers. This finding suggests that if the source of negative social interactions is someone important, the adolescents will feel more vulnerable and stressful. The current study measured relations with parents, teachers, and peers, all of whom are important to adolescents. The fact that Taiwanese adolescents appraise negative social relations as a serious source of stress may be one contributing factor to explain why negative social relations were found to be significant in the current study. However, more studies are needed to clarify this speculation, and to investigate the extent to which the findings are generalizable to other cultures.

There are some additional limitations to the present study. First, as the study focused on adolescents, we do not know if the current findings are applicable to adults or younger children. Indeed, there may be differences in psychological strength and social network complexity across different age groups. Second, to focus on exploring the roles of social relations, we did not include earthquake-exposure variables in the models. One of our other studies (Chen et al., 2002) had found dose-effect among earthquake exposure and PTSD symptoms in adolescents in the first year after the Chi-Chi Earthquake. However, without earthquakerelated stresses in the models of current study, we cannot clarify the effects of earthquake exposure. Another limitation is that some people, though not a big portion, moved out of the earthquakeaffected area. Thus, the sample used in this study may not be representative of all victims from the epicenter.

In conclusion, the current study suggests the importance of taking detrimental social relations into consideration in dealing with the issue of social support after trauma. When working with trauma-exposed adolescents, especially those who are likely or 
partially diagnosed with PTSD, it is important to help them reduce their exposure to detrimental social relations. For example, assisting significant others in enhancing their supportive skills may be quite advantageous for these adolescents. In this regard, it has been found in clinical settings that having the child and their parents join in therapy sessions enhances relief of childhood traumatic grief (Cohen, Mannarino, \& Knudsen, 2004). In addition, Scheeringa and Zeanah (2001) have pointed out that a caregiver's own trauma or response to a child's trauma may interfere with her or his ability to provide appropriate care and support. They have also suggested that helping a significant other to deal with their own trauma may be critical to enhancing their ability to provide support to promote the recovery of children and adolescents after trauma.

\section{REFERENCES}

American Psychiatric Association. (1994). Diagnostic and statistical manual of mental disorders (4th ed.). Washington, DC: Author.

Andrews, B., Brewin, C. R., \& Rose, S. (2003). Gender, social support and PTSD in victims of violent crime. Journal of Traumatic Stress, 16, 421-427.

Brewin, C. R., Andrews, B., \& Valentine, J. D. (2000). Meta-analysis of risk factors for post-traumatic stress disorder in trauma exposed adults. Journal of Consulting and Clinical Psychology, 68, 748-766.

Chen, S. H., Lin, Y. H., Tseng, H. M., \& Wu, Y. C. (2002). Posttraumatic stress reactions in children and adolescents one year after the 1999 Taiwan Chi-Chi Earthquake. Journal of the Chinese Institute of Engineers, 25, 597-609.

Chen, S. H., \& Wu, Y. C. (2006). Changes of PTSD symptoms and school reconstruction: A two-year prospective study of children and adolescents after the Taiwan 921 Earthquake. Nature Hazards, 37, 225-244.

Cohen, J. A., Mannarino, A. P., \& Knudsen, K. (2004). Treating childhood traumatic grief: A pilot study. Journal of the American Academy of Child and Adolescent Psychiatry, 43, 1225-1233.

Dohrenwend, B. (2000). The role of adversity and stress in psycholpathology: Some evidence and its implications for theory and research. Journal of Health and Social Behavior, 41, 1-19.

Guay, S., Billette, V., \& Marchand, A. (2006). Exploring the links between posttraumatic stress disorder and social support: Processes and potential research avenues. Journal of Traumatic Stress, 19, 327-338.

Hobfoll, S. E., \& Stephens, M. A. P. (1990). Social support during extreme stress: Consequences and intervention. In B. R. Sarason, I. G. Sarason, \& G. R. Pierce (Eds.), Social support: An interactional view (pp. 454-481). New York: Wiley.

Hu, L. T., \& Bentler, P. M. (1999). Cutoff criteria for fit indexes in covariance structure analysis: Conventional criteria versus new alternatives. Structural Equation Modeling, 6, 1-55.

Joreskog, K., \& Sorbom, D. (2004). LISREL (Version 8.7) [Computer software]. Lincolnwood, IL: Scientific Software International.

Kaniasty, K. (2005). Social support and traumatic stress. PTSD Research Quarterly, $16,2,1-7$

Kaniasty, K. Z., \& Norris, F. H. (2008). Longitudinal linkages between perceived social support and posttraumatic stress symptoms: Sequential roles of social causation and social selection. Journal of Traumatic Stress, 21, 274-281.

Kaniasty, K. Z., Norris, F. H., \& Murrell, S. A. (1990). Received and perceived social support following natural disaster. Journal of Applied Social Psychology, 20, 85-114.
King, D., Taft, C., King, L., Hammond, C., \& Stone, E. (2006). Directionality of the association between social support and posttraumatic stress disorder: A longitudinal investigation. Journal of Applied Social Psychology, 36, 29802992.

Kline, R. B. (2005). Principles and practice of structural equation modeling. New York: Guilford Press.

Kupersmidt, J. B., \& Coie, J. D. (1990). Preadolescent peer status, aggression and school adjustment as predictors of externalizing problems in adolescence. Child Development, 61, 1350-1362.

MacKinnon, D. P., Lockwood C. M., Hoffman, J. M., West, S. G., \& Sheets, V. (2002). A comparison of methods to test the significance of mediation and other intervening variable effects. Psychological Methods, 7, 83-104.

National Alliance for Post-Earthquake Reconstruction. (2000). Q and A: Post 921 earthquake reconstruction/Q and A: Post 921 rehabilitation 1. Taipei, Taiwan: Author.

Norris, F. H., Friedman, M. J., Watson, P. J., Byrne, C. M., Diaz, E., \& Kaniasty, K. (2002). 60,000 Disaster victims speak: Part I. An empirical review of the empirical literature, 1981-2001. Psychiatry: Interpersonal \& Biological Processes, 65, 207-239.

Ozer, E. J., Best, S. R., Lipsey, T. L., \& Weiss, D. S. (2003). Predictors of posttraumatic stress disorder and symptoms in adults: A metaanalysis. Psychological Bulletin, 129, 52-73.

Pine, D. S., \& Cohen, J. A. (2002). Trauma in children and adolescents: Risk and treatment of psychiatric sequelae. Biological Psychiatry, 51, 519-531.

Pfefferbaum, B., Stuber, J., Galea, S., \& Fairbrother, G. (2006). Panic reactions to terrorist attacks and probable posttraumatic stress disorder in adolescents. Journal of Traumatic Stress, 19, 217-228.

Pynoos, R., Steinberg, A., \& Wraith, R. (1995). A developmental model of childhood traumatic stress. In D. Cicchetti \& D. J. Cohen (Eds.), Developmental psychopathology (Vol. 2, pp. 72-95). New York: Wiley.

Rook, K. S. (1998). Investigating the positive and negative sides of personal relationships: Through a lens darkly? In B. H. Spitzberg \& W. R. Cupach (Eds.), The dark side of close relationships (pp. 369-393). Mahwah, NJ: Erlbaum.

Rook, K. S. (2003). Exposure and reactivity to negative social exchanges: A preliminary investigation using daily diary data. Journal of Gerontology: Psychological Sciences, 58, 100-111.

Scheeringa, M. S., \& Zeanah, C. H. (2001). A relational perspective on PTSD in early childhood and adolescents. Journal of Traumatic Stress, 14, 799-815.

Schneider, B. H. (2000). Friends and enemies: Peer relations in childhood. London: Arnold.

Schuster, T. L., Kessler, R. C., \& Aseltine, R. H., Jr. (1990). Supportive interactions, negative interactions, and depressed mood. American Journal of Community Psychology, 18, 423-438.

Steinberg, A. M., Brymer, M., Decker, K., \& Pynoos, R. S. (2004). The UCLA PTSD Reaction Index. Current Psychiatry Reports, 6, 96-100.

Wu, C. H., Wu, Y. C., Hsu, W. Y., \& Shiau, R. J. (2008). Development of the Taiwan Relationship Inventory for Children and Adolescents (TRICA) and its psychometric study. The Chinese Journal of Psychological Testing, 55, 535557.

Yang, K. S. (1981). Social orientation and individual modernity among Chinese students in Taiwan. Journal of Social Psychology, 113, 159-170.

Yeh, P. C., \& Lay, K. L. (2008). Children's vulnerability and inflammability to peer verbal provocation: Sociometric and developmental differences. Chinese Journal of Psychology, 50, 49-69.

Zoellner, L. A., Foa, E. B., \& Bartholomew, D. B. (1999). Interpersonal friction and PTSD in female victims of sexual and nonsexual assault. Journal of Traumatic Stress, 12, 689-700. 